\title{
Exudative epidermitis in Iraqi camels
}

\author{
M. H. Hussain \\ F. G. Habasha
}

Coll. of Vet. Med/ Univ. of Al-Qadissiya Coll. of Vet. Med./ Univ. of Baghdad

\section{Abstract}

The survey began in December 2008 \& finished in June 2009 in three governorates; AlQadissiya, Al-Najaf \& Al-Muthanna in different locations inspecting 2412 dromedaries, we found out bacterial affections, samples were carried out to detect the exudative epidermitis with Staphylococcus hyicus in 213 camels which present $8.8 \%$ of the total inspected camels. There was an obvious age-depending infection; exudative epidermitis diagnosed in $74.2 \%$ in camels ranged in 5-10 years old, $15 \%$ in camels less than 5 years old \& $10.8 \%$ in camels more than 10 years old.

\section{Introduction}

Staphylococcosis is the most common infection of skin (1). Gram positive aerobic cocci are conveniently divided into groups based on catalase production. The family Micrococcaceae including Staphylococcus, Micrococcus and Rothia (Stomatococcus) are catalase positive, whereas the genera Streptococcus, Enterococcus, Eremococcus, Gemella, Globicatella and Vagococcus are catalase negative. Staphylococci is the most commonly isolated Micrococcaceae from veterinary clinical specimens. Microscopically; they occur in pairs, grapelike clusters or singly, colonies are generally white to off-white, with smooth surface and butyrous consistency, genus staphylococcus can be usually differentiated from micrococcus based on cell morphology; the later form tetrads and cells tend to be larger than those of the staphylococcus and pigment production on solid media (3).Staphylococcus hyicus is reported to be an important cutaneous pathogen in animals in addition to its presence in cases like arthritis in pigs and

\section{Materials \& methods}

I- Materials

$\checkmark$ Manitol salt agar

$\checkmark$ Brain heart infusion agar

$\checkmark$ Brain heart infusion broth

$\checkmark$ hydrogen peroxide

$\checkmark$ rabbit plasma

$\checkmark$ API staph kits

\section{II- Animals of the study:}

Three governorates were involved in this research Al-Qadissiya, Al-Najaf and Al-Muthanna, samples collected from the mastitis in cattle. The eighth edition of Bergey's manual doesn't list $S$. hyicus as a separate species, instead classifying it as biotype 2 of $S$. epidermidis (albus). Nowadays the name $S$. hyicus is widespread usage in veterinary literature. There are two subspecies of $S$. hyicus; $S$. hyicus subsp. hyicus and S. hyicus subsp. Chromogenes, an organism of questionable virulence frequently isolated from mastitic milk (4). For diagnosis, primary culture for Staphylococcus spp. is best accomplished on blood agar and selective media like manitol salt agar and staph 110 agar. Coagulase testing and determination of hemolytic pattern are important initial tests. Rapid commercial methods are available for identification of most of Staphylococcus spp. (4).S. hyicus has been found in pigs, cattle and cow's milk, it's associated with exudative epidermitis, an acute disease of suckling weaned pigs (2). Omer and Eltinay has isolated S. hyicus from camels' raw milk in central and southern regions of UAE in 2008.

\section{$\checkmark$ Gram stain \\ $\checkmark$ Oxidase reagent \\ $\checkmark$ Catalase reagent \\ $\checkmark$ Blood agar base \\ $\checkmark$ McConkey agar \\ $\checkmark$ Nutrient agar \\ $\checkmark$ Nutrient broth}

slaughter houses, sale yards and the herds found in several locations randomly. The research began on December 2008 and finished on June 2009. 2412 Iraqi 
dromedaries were examined regardless to location, age, sex and breed, from which 213 camels were suffering exudative epidermitis.213 infected camels were examined and filmed with exudative skin lesions that were multifocal on the chest, neck, abdomen and thigh respectively. Transient media were used, nutrient broth and/ or brain heart infusion broth, to keep the samples fresh and out of contaminations. Steps used for making an accurate diagnosis of the pathogen causing this exudative lesions were:-

A- Morphological identification which depends on the direct gram staining smear in which the cocci shape were filmed, making culture of the samples in order to obtain the colony on different media; nutrient agar, brain-heart infusion agar , MacConkey and blood agar. Purification cultures were done other times to get pure colonies on the Brain-heart infusion agar and Blood agar. Smears were taken from the pure colonies to be stained with a gram stain which has shown mostly a staphylococcal shape positively.

$\boldsymbol{B}$ - Biochemical reactions:

1- Catalase tests were done by adding few drops of $3 \%$ hydrogen peroxide on a clean slide which mixed with pure colony (not from blood agar) that held by a sterile loop, small bulbs were seen within few minutes giving a positive reaction.

2- Oxidase test was also done by Placing a piece of filter paper on a clean microscope slide, 2 to 3 drops of oxidase reagent placed onto the filter paper. An isolated colony was touched with a wooden applicator stick and the end of the stick was placed, carrying some of the isolate, onto the reagent saturated filter paper. Then, the presence of a dark purple color was observed, the reaction is positive if the smear turns purple within 10-30 seconds.

3- Samples examined with coagulase test using rabbit plasma gave a negative reaction that means they were nonaureus staphylococci. Add $0.5 \mathrm{ml}$ reconstituted lyophilized rabbit coagulase plasma with EDTA to a sterile tube, touch an isolated colony with an inoculating loop, place the loop, carrying some of the isolate, into the tube containing the rabbit plasma, and mix thoroughly. Incubate the tube at $35^{\circ}$ $\mathrm{C}$ for 6 hours observing the tube for the presence of clotting. If no clotting is observed, incubate for 24 hours and observe again. The reaction is positive if clotting is present (The plasma will gel to a viscosity where it will not flow down the tube when tilted at a $45^{\circ}$ angle) (5).

C- $\quad$ API Staph (Analytical Profile Index)Samples were sent to the Central Public Health Laboratories cultured on Brain-heart infusion agar to be diagnosed using technique of API staph kits. Analytical tests were done before this diagnosis; all samples were positive to catalase test, negative to oxidase and coagulase tests. The results were documented as staphylococcus hyicus.

20 microtubes containing dehydrated substrates (formerly API staph-Trac,bioMerieux- vitek, Inc.). These microtubes are inoculated with a bacterial suspension prepared in API Staph medium that reconstitutes the test. During incubation, metabolism produces color changes that are either spontaneous or revealed by the addition or reagents. The reactions are read according to the reading table and the identification is obtained by referring to the analytical profile index or using the identification software.

\section{Results \& discussion}

213 camels in different ages and sexes, showed exudative epidermitis with Staphylococcus hyicus. Creamy to white exudative discharge, as in fig(1), found out of skin that may be swelled as a node shape up to $3 \mathrm{~cm}$ in diameter filled with exudate or it is difficulty palpable in the early stages. Exudates were multifocal lesions spread on the chest, neck, abdomen, and thigh respectively, table (1) shows that camels in 5-10 years infected with $74.2 \%, 15 \%$ in camels less than 5 years and $10.8 \%$ in camels more than 10 years old. Female has the higher percentage of infection $60.09 \%$ and that may be due to the large proportion of females in camel herds which may reach 
99\%.Location has no effect on the distribution of the disease especially that the three governorates have the same weather. Sampling the exudates has found out the bacterial and colony shape in the laboratory, fig (2) shows the colony shape that are circular, (0.5-1.5)mm, creamy, white, convex, and non-hemolytic bacterium, fig(3) shows the microscopic appearance of gram positive bacteria in slide stained with gram stain in which the bacteria arranged in grape-like clusters.

Table (1): Numbers of infected camels with exudative epidermitis according to the location, age and sex

\begin{tabular}{|c|c|c|c|c|c|c|c|c|}
\hline \multirow{2}{*}{\multicolumn{2}{|c|}{ Locations }} & \multirow{2}{*}{$\begin{array}{c}\text { Infected } \\
\text { camels }\end{array}$} & \multicolumn{2}{|c|}{$\sim 5$ years } & \multicolumn{2}{|c|}{$5-10$ years } & \multicolumn{2}{|c|}{ 10 years } \\
\hline & & & male & female & male & Female & Male & female \\
\hline \multirow{6}{*}{$\begin{array}{c}\text { Al- } \\
\text { Qadissyia }\end{array}$} & Slaughter house & 33 & 0 & 0 & 6 & 19 & 5 & 3 \\
\hline & $\begin{array}{l}\text { Al-Hamza Al- } \\
\text { Shargy }\end{array}$ & 12 & 0 & 3 & 1 & 7 & 1 & 0 \\
\hline & Al-Daghara & 41 & 2 & 7 & 1 & 30 & 1 & 0 \\
\hline & Aal-Bdeer & 42 & 4 & 12 & 4 & 20 & 1 & 1 \\
\hline & Al-Shaf'ya & 0 & 0 & 0 & 0 & 0 & 0 & 0 \\
\hline & Al-Shenafeya & 21 & 0 & 0 & 1 & 20 & 0 & 0 \\
\hline \multirow{2}{*}{ Al-Najaf } & Slaughter house & 22 & 0 & 0 & 7 & 9 & 4 & 2 \\
\hline & Sale yard & 17 & 2 & 2 & 6 & 3 & 3 & 1 \\
\hline \multirow{3}{*}{$\begin{array}{c}\text { Al- } \\
\text { Muthanna }\end{array}$} & Slaughter house & 8 & 0 & 0 & 3 & 4 & 1 & 0 \\
\hline & Sale yard & 0 & 0 & 0 & 0 & 0 & 0 & 0 \\
\hline & Herds & 17 & 0 & 0 & 1 & 16 & 0 & 0 \\
\hline \multicolumn{2}{|l|}{ Total } & 213 & 8 & 24 & 30 & 128 & 16 & 7 \\
\hline \multicolumn{2}{|l|}{ Percentage } & & 3.76 & 11.2 & 14.09 & 60.09 & 7.51 & 3.29 \\
\hline \multicolumn{2}{|l|}{ Total } & & \multicolumn{2}{|c|}{32} & \multicolumn{2}{|c|}{158} & \multicolumn{2}{|c|}{23} \\
\hline \multicolumn{2}{|l|}{ Percentage } & & \multicolumn{2}{|c|}{15} & \multicolumn{2}{|c|}{74.2} & \multicolumn{2}{|c|}{10.8} \\
\hline
\end{tabular}

The bacteria show catalase positive test, oxidase negative and coagulase negative test. API staph is an 18-24 hr identification system for both micrococci and staphylococci. This system contains 20 microtubes tests arranged in a strip form and was inoculated with a suspension of the organism prepared in API staph medium provided with the kit. After reading the biochemical reactions from the change in colors as in fig (4), a seven-digit octal code was generated and identification of the organism was obtained with the computerassisted data base. Central Public Health Laboratories had documented the diagnosis by using the result sheet of API staph giving the genus staphylococcus hyicus.

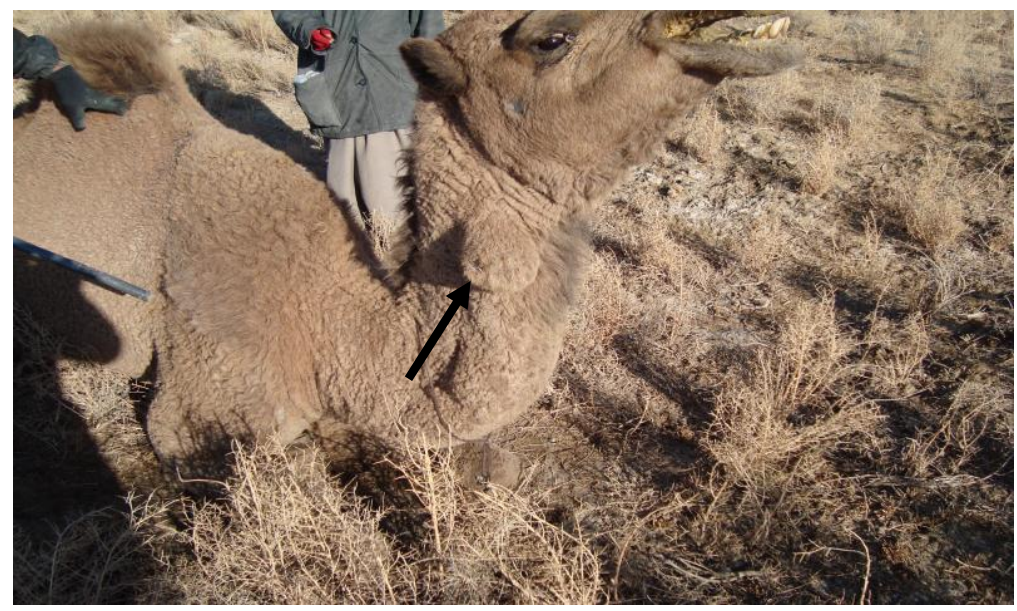

Fig (1): Exudative epidermitis in 6 years she-camel with exudate-filled node on the neck. 


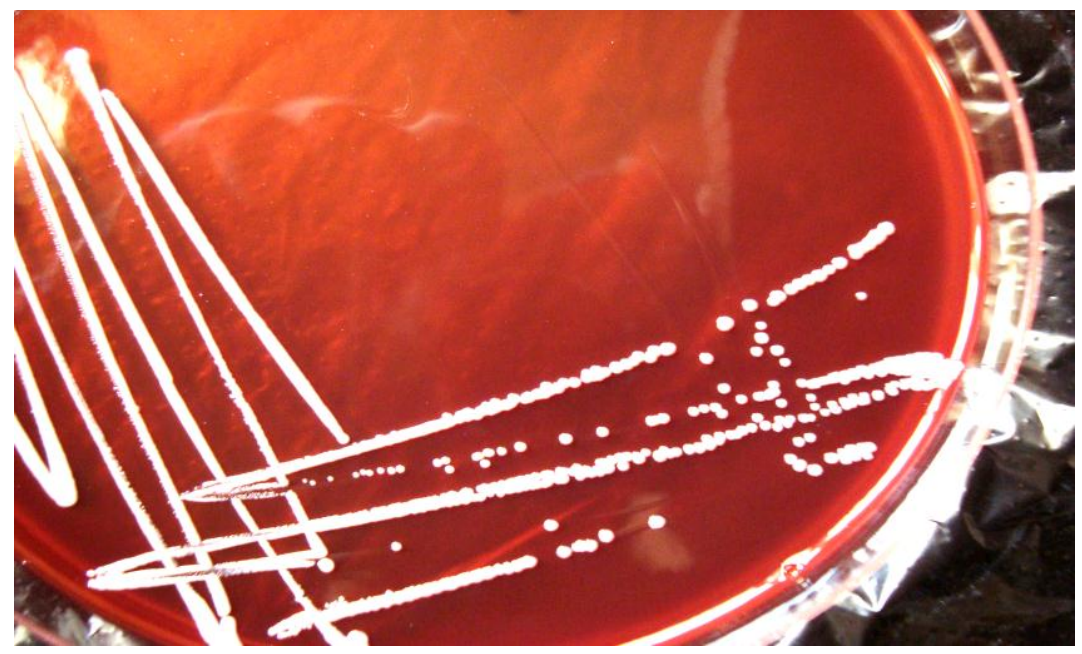

Fig (2): Colony shape of staphylococcus hyicus on blood agar after 48hr. with no hemolysis.

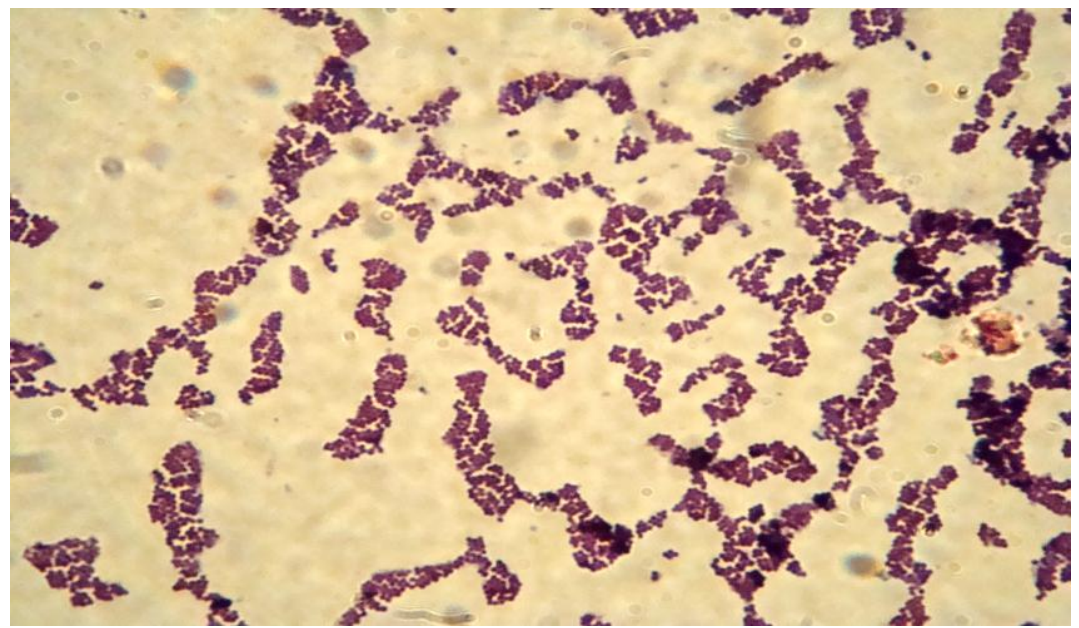

Fig (3): Microscopic appearance of gram positive staphylococcus hyicus shows the grape-like clusters.X100

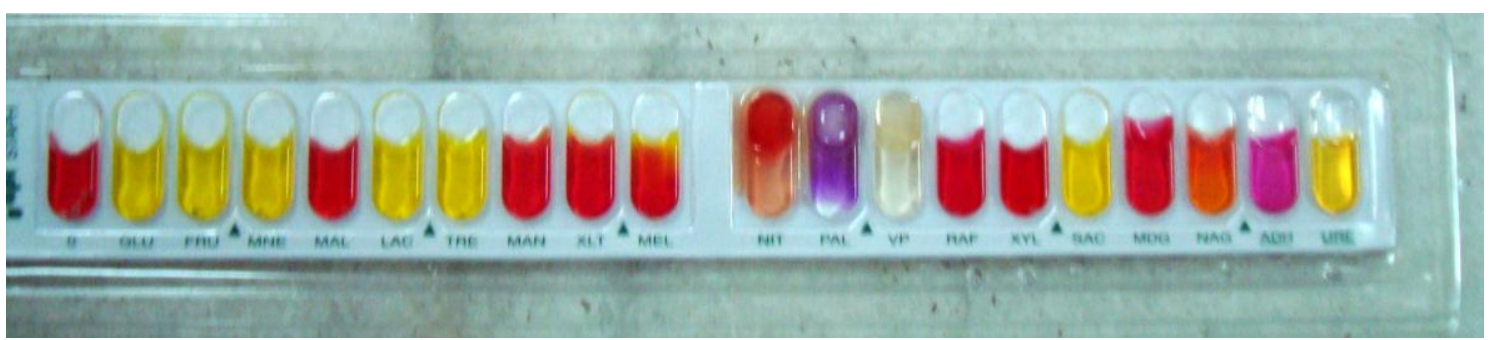

Fig (4): API staph strip after $22 \mathrm{hr}$. incubation

Camels having Exudative epidermitis infection show no systemic reactions; infebrile $(36.3 \pm 0.03) \mathrm{C}^{\mathbf{o}}$, respiratory rate about $(15 \pm 0.5)$ per minute, $(45 \pm 1.5)$ pulse rate per minute, no hair lose in the site of lesions no lameness when infection affects the legs, good appetite, good rumination, pink mucous membrane, no itching and in several cases there were accidental respiratory infections and cough or diarrhea.Exudative epidermitis has reported in all ages in the study which makes it the most common disease. Besides, owners don't interest with this disease due to their 
medical fallen awareness, this disease needs a long and expensive medicines which make it difficult especially within Iraqi poor bedouins. Another cause that this disease doesn't result in systemic reaction on the camels which makes it sometimes unnoticeable. No previous reports had published about infection with Exudative epidermitis in camels except the presence of "greasy pigs" as it called in pigs.

\section{References}

1. Brooks, G.F.; Butel, J.S., \& Morse, S.A. (1998). Jawetz Melnick \& Adelbergs Medical microbiology. $21^{\text {st }}$ ed. Appelton \& Lange. California. pp:216220,372-379.

2. Devriese, L.A.; Hajek, V.; Oeding, P.; Meyer, S.A.; \& Schleifer, K.H.(1978). Staphylococcus hyicus (Sompolinsky, 1953) Comb. Nov.\& Staphylococcus hyicus subsp.chromogenes subsp. Nov. Int J. Syst. Bacterial. 28:482-490.

3. Elmer, W.K.; Stephen, D.A.; William, M.J.; \& Paul, C.S. (1997). Color atlas \& textbook of diagnostic microbiology. $\quad 5^{\text {th }}$ edition, Lippincott Williams \& Wilkins, Philadelphia, USA.
4. John, F.T.; James, H.G.; Fredric, W.S.; \& Jeffrey, E.B. (1988). Hagan \& Bruner's Microbiology \& Infectious Diseases of Domestic Animals. The genera Staphylococcus spp.. $8^{\text {th }}$ edition. Comstock Publishing Associates, Cornell University Press, London.

5. Macfaddin, J.E.(2000). Biochemical tests for identification of medical becteria. 3rd ed. Lippincott Williams and Wilking. England, UK.

6. Omer, R.H.; \& Eltinay, A.H. (2008). Microbial quality of camels' raw milk in central \& southern regions of United Arab Emirates. Emir. J. Food Agric. 2008. 20(1):76-83.

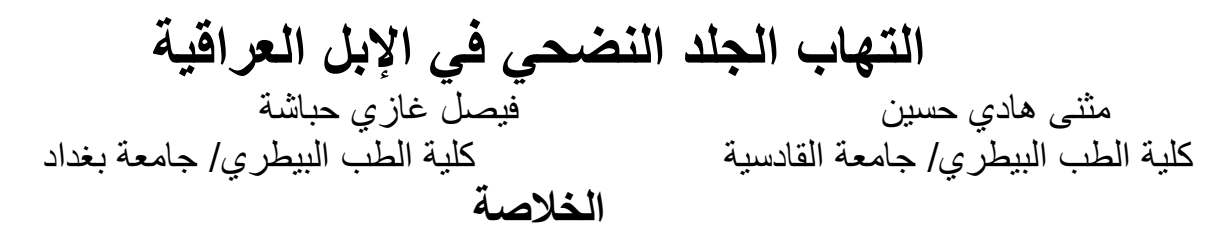

بدأت هذه الدراسة في كانون الأول 2008 وانتهت في حزيران 2009 في مواقع مختلفة من ثلاث محافظات;

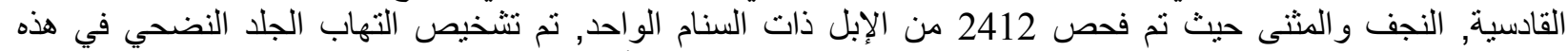

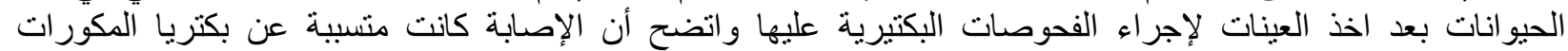

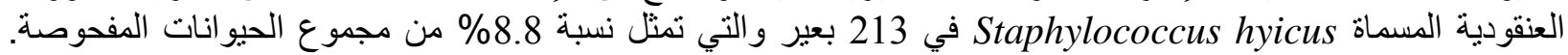

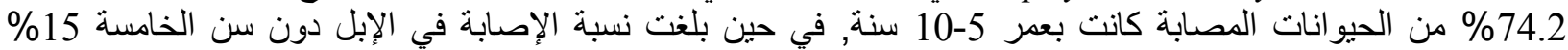

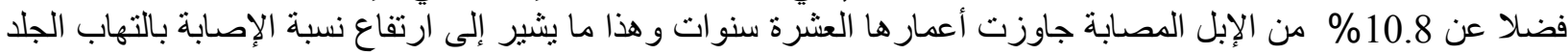

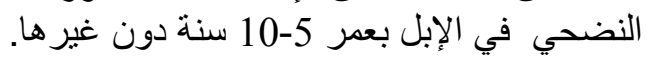

